\title{
GMR
}

\section{Inhibition of Atg6 and Pi3K59F autophagy genes in neurons decreases lifespan and locomotor ability in Drosophila melanogaster}

\author{
P.G. M'Angale and B.E. Staveley \\ Department of Biology, Memorial University of Newfoundland, St. John's, \\ Newfoundland and Labrador, Canada \\ Corresponding author: B.E. Staveley \\ E-mail: bestave@mun.ca
}

Genet. Mol. Res. 15 (4): gmr15048953

Received July 7, 2016

Accepted August 23, 2016

Published October 24, 2016

DOI http://dx.doi.org/10.4238/gmr15048953

Copyright $(2016$ The Authors. This is an open-access article distributed under the terms of the Creative Commons Attribution ShareAlike (CC BY-SA) 4.0 License.

\begin{abstract}
Autophagy is a cellular mechanism implicated in the pathology of Parkinson's disease. The proteins Atg6 (Beclin 1) and Pi3K59F are involved in autophagosome formation, a key step in the initiation of autophagy. We first used the GMR-Gal4 driver to determine the effect of reducing the expression of the genes encoding these proteins on the developing Drosophila melanogaster eye. Subsequently, we inhibited their expression in D. melanogaster neurons under the direction of a Dopa decarboxylase $(D d c)$ transgene, and examined the effects on longevity and motor function. Decreased longevity coupled with an age-dependent loss of climbing ability was observed. In addition, we investigated the roles of these genes in the well-studied $\alpha$-synuclein-induced Drosophila model of Parkinson's disease. In this context, lowered expression of Atg6 or Pi3K59F in $D d c$-Gal4-expressing neurons results in decreased longevity and associated age-dependent loss of locomotor ability. Inhibition of Atg6
\end{abstract}

Genetics and Molecular Research 15 (4): gmr15048953 
or Pi3K59F together with overexpression of the sole pro-survival $\mathrm{Bcl}-2$ Drosophila homolog Buffy in Ddc-Gal4-expressing neurons resulted in further decrease in the survival and climbing ability of Atg6-RNAi flies, whereas these measures were ameliorated in Pi3K59F-RNAi flies.

Key words: Atg6; Beclin 1; Pi3K59F; Buffy; $\alpha$-synuclein; Parkinson's disease

\section{INTRODUCTION}

Parkinson's disease (PD) is the second most common human neurodegenerative condition, and is characterized by progressive degeneration and loss of dopamine-producing neurons in the centrally located substantia nigra pars compacta region of the brain. PD is marked by severe locomotor dysfunction, as well as non-motor symptoms such as autonomic, cognitive, and psychiatric deficiencies (Forno, 1996). Although the majority of PD cases are sporadic, familial forms with a genetic component have been identified and studied extensively in model organisms (Staveley, 2014). The first gene to be associated with familial PD encodes $\alpha$-synuclein, a small soluble protein predominantly found in neural tissues (Polymeropoulos et al., 1997; Dehay et al., 2016). The association of $\alpha$-synuclein with mitochondrial components is thought to lead to oxidative stress, apoptosis, autophagy, and ultimately, neurodegeneration (Chinta et al., 2010; Esteves et al., 2011). A link between autophagic dysfunction and aberrant $\alpha$-synuclein has been established in the pathogenesis of both sporadic and familial forms of PD (Cuervo et al., 2004). PD pathogenesis must therefore involve the failure of several cellular mechanisms, with the disruption of autophagic homeostasis contributing to the development of this neurodegenerative disorder.

Autophagy is a tightly regulated catabolic mechanism in eukaryotic cells that degrades long-lived proteins and organelles. It comprises a multi-step pathway involving key regulatory factors such as TOR kinase, AMP-activated protein kinase, $\mathrm{Bcl}-2 / \mathrm{Bcl}-\mathrm{X}_{\mathrm{L}}$ inhibition of the Beclin 1/class III PI3K complex, and p53 tumor suppressor protein, among others, that respond to energy levels and the absence or presence of nutrients and growth factors (Levine and Kroemer, 2008; Xilouri and Stefanis, 2011). The initiation of autophagy is marked by phagophore formation, in which the ULK1-Atg13-FIP200 complex is pivotal. The nucleation phase of early phagophore formation requires interaction between the ULK1 complex and the Atg6-interacting complex, composed of Atg6, class III PI3K, Vps15, and Atg14L. Stimulation of this complex generates phosphatidylinositol-3-phosphate, which promotes autophagosomal membrane nucleation (Levine and Kroemer, 2008). This is followed by elongation and maturation of the autophagosome, before it finally fuses with an acidic lysosome.

The Atg6-interacting complex acts in a regulatory step, with autophagy being promoted by Atg6-binding proteins such as AMBRA1, UVRAG, and Bif-1, and inhibited by Rubicon and Bcl-2/Bcl- $\mathrm{X}_{\mathrm{L}}$. Inhibition of Atg6-dependent autophagy by anti-apoptotic Bcl-2 proteins implies a regulatory role for this important protein family (Pattingre et al., 2005). One mechanism thought to be responsible for such inhibition involves the binding of antiapoptotic Bcl-2 proteins to the Bcl-2 homology 3 (BH3) domain of Atg6, thus interfering with the initiation of autophagy. One group has suggested that this protein family affects autophagy only indirectly, through inhibition of the pro-apoptotic Bcl-2 proteins Bax and Bak (Lindqvist et al., 2014). The sequestration of Atg6 by Bcl-2 proteins can be reversed by phosphorylation

Genetics and Molecular Research 15 (4): gmr15048953 
of the former's BH3 domain by death-associated protein kinase (Zalckvar et al., 2009). Atg6 contains the APG6 domain that binds class III PI3K to affect its role in vacuolar protein sorting and autophagy (Furuya et al., 2005). Autophagy is highly conserved, with single orthologs of many of the components involved in this mechanism present in Drosophila melanogaster (McPhee and Baehrecke, 2009; Zirin and Perrimon, 2010). For example, the Atg6-interaction complex seems to be well conserved and is central to autophagy (Juhász et al., 2008; Chang and Neufeld, 2010). Orthologs of Atg14L, UVRAG, Rubicon, and Bcl-2 are present in the fly and may play the same roles as their mammalian counterparts by forming various autophagy regulatory complexes.

PD counts among those diseases in which the dysregulation of autophagy has been implicated. Their vesicular nature and the dominant pathological features of surviving neurons in patients with PD has led to the suggestion that the autophagy-lysosome pathway contributes to the formation or dissolution of Lewy bodies (LBs) (Perrett et al., 2015; Xilouri and Stefanis, 2015). The implication of autophagy in human disease, especially in neurodegenerative disorders, makes Drosophila an attractive model to study the consequences of altering genes involved in this process, such as Atg6 and the class III PI3K Pi3K59F. The first fly model of PD utilized a human $\alpha$-synuclein transgene to induce PD-like symptoms (Feany and Bender, 2000). This model has become one of the most successful systems for the study of this disease, owing to accurate replication of the degeneration and loss of DA neurons, age-dependent loss of locomotor ability, presence of LB-like inclusions, and compromised survival (Staveley, 2014). We determined the effect of Atg6 or Pi3K59F inhibition via directed expression of stable RNA interference (RNAi) transgenes in Dopa decarboxylase (Ddc)-Gal4-expressing Drosophila neurons. Furthermore, these transgenes were co-expressed with $\alpha$-synuclein to investigate the role of Atg6 and Pi3K59F in the original Drosophila model of PD. In addition, loss of function of these autophagy genes was evaluated in the context of overexpression of Buffy, the only pro-survival Bcl-2 homolog in Drosophila.

\section{MATERIAL AND METHODS}

\section{Bioinformatic analysis}

Protein sequences were obtained from the National Center for Biotechnology Information (NCBI; http://www.ncbi.nlm.nih.gov/protein), and domains were identified using the NCBI Conserved Domain Database (http://www.ncbi.nlm.nih.gov/cdd; Marchler-Bauer et al., 2015) and the Eukaryotic Linear Motif (ELM) resource (http://elm.eu.org; Dinkel et al., 2016), which focuses on annotation and detection of ELMs, also known as short-linear motifs. Clustal Omega (http://www.ebi.ac.uk/Tools/msa/clustalo; Goujon et al., 2010; Sievers et al., 2011) was used for multiple-sequence alignment and to identify the conservation of domains across selected organisms. The presence of nuclear export signals (NESs) was predicted using the NetNES 1.1 server (http://www.cbs.dtu.dk/services/NetNES; la Cour et al., 2004).

\section{Drosophila stocks and media}

The UAS-Atg6-RNAi ( $\left.w^{\text {Ills }} ; \quad P\{G D 11647\} v 22122\right)$ and UAS-Pi3K59F-RNAi $(P\{K K 107602\}$ VIE-260B) lines were obtained from the Vienna Drosophila Resource Center (Vienna, Austria). UAS- $\alpha$-synuclein flies (Feany and Bender, 2000) were generously provided

Genetics and Molecular Research 15 (4): gmr15048953 
by Dr. M. Feany of the Harvard Medical School, UAS-Buffy flies (Quinn et al., 2003) by Dr. L. Quinn of the University of Melbourne, and Ddc-Gal4 flies (Li et al., 2000) by Dr. J. Hirsch of the University of Virginia. GMR-Gal4 (Freeman, 1996) and UAS-lacZ flies were obtained from the Bloomington Drosophila Stock Center at Indiana University, IN, USA. Stocks and crosses were maintained on standard cornmeal/molasses/yeast/agar medium treated with propionic acid and methylparaben to inhibit fungal growth. Stocks were maintained at room temperature $\left(22^{\circ} \pm 3^{\circ} \mathrm{C}\right)$, whereas crosses and experiments were carried out at $25^{\circ}$ and $29^{\circ} \mathrm{C}$.

\section{Derivative Drosophila lines}

$U A S-\alpha$-synuclein/CyO; Ddc-Gal4/TM3 and UAS- $\alpha$-synuclein/CyO; GMR-Gal4 flies, in which $\alpha$-synuclein was overexpressed in neurons under the direction of Ddc-Gal4 and the developing eye under the control of GMR-Gal4, respectively, were generated according to standard homologous recombination methods. The UAS-Buffy/CyO; Ddc-Gal4 and UASBuffy/CyO; GMR-Gal4 lines were used to overexpress Buffy in neurons and the developing eye, respectively. Polymerase chain reaction (PCR) was employed to detect the presence of the $\alpha$-synuclein construct using primers designed with the NCBI primer design tool according to the Homo sapiens synuclein, alpha (non-A4 component of amyloid precursor), transcript variant 1 mRNA sequence (NCBI reference sequence NM_000345.3). The 5'- to 3'-sequence of the forward primer was GTGCCCAGTCATGACATTT, and that of the reverse primer was CCACAAAATCCACAGCACAC; both primers were synthesized by Invitrogen (Carlsbad, CA, USA). The D. melanogaster Buffy mRNA sequence (NM_078978.2) was used to design a set of primers targeting the endogenous gene and transgenic construct. The 5'- to 3'-sequences of the forward primers were CACAGCGTTTATCCTGCTGA and CGGGTGGTGAGTTCCATACT, while those of the reverse primers were TCGCAGTGTGAAGATTCAGG andTTAATCCACGG AACCAGCTC; these primers were ordered from Eurofins MWG Operon (Louisville, KY, USA). Gel electrophoresis was performed for confirmation of recombination events based on the presence of the corresponding PCR product.

\section{Survival assay}

Fly survival was assessed following a standard protocol, as previously described (Todd and Staveley, 2012; M'Angale and Staveley, 2016). Briefly, over 200 flies were monitored per genotype and scored every 2 days for the presence of deceased adults (Staveley et al., 1990). Longevity data were analyzed using GraphPad Prism version 5.04 (GraphPad Software, Inc., La Jolla, CA, USA), and survival curves were compared by the log-rank (Mantel-Cox) test. Significance was determined at a 95\% confidence level (P $\leq$ $0.05)$ with Bonferroni correction.

\section{Climbing assay}

The climbing assay was performed as previously described, and repeated three times (Todd and Staveley, 2004). Climbing indices were computed and subsequently analyzed using GraphPad Prism version 5.04. The 5-climbing index is a model employed for graded climbing analysis using non-linear regression, " 5 " being the highest level that flies can climb. Data were compared using $95 \%$ confidence intervals (CI) and a P value threshold of 0.05 .

Genetics and Molecular Research 15 (4): gmr15048953 


\section{Scanning electron microscopy of Drosophila eyes}

Drosophila eyes were prepared for scanning electron microscopy following a standard protocol, as previously described (M'Angale and Staveley, 2016). For each cross, at least 10 different images of eyes from adult flies 5 days post-eclosion per genotype were analyzed using the ImageJ software (National Institutes of Health; Schneider et al., 2012), and biometric analysis was performed using GraphPad Prism version 5.04. The relative size of the disrupted eye area was calculated as detailed in a previous publication (M'Angale and Staveley, 2012). Statistical comparisons comprised one-way analyses of variance and Dunnett multiple comparison tests. $\mathrm{P}$ values less than 0.05 were considered significant.

\section{RESULTS}

\section{Atg6 and Pi3K59F are evolutionarily conserved}

Bioinformatic analysis of Atg6 (also known as Beclin 1) and Pi3K59F protein sequences revealed remarkable conservation of domains and highlights the importance of these two autophagy proteins (Figure 1). The Drosophila Atg6 protein consists of 422 amino acids, and shares $50 \%$ identity and $67 \%$ similarity with human Beclin 1, comprising 450 amino acids, alignment with which revealed conserved APG6/Vps30 and NES domains (Figure 1A). A putative non-canonical BH3 domain appears to be present in the Drosophila sequence that may function in Atg6 inhibition through sequestration by the pro-survival Bcl-2 proteins, as occurs in mammals (Figure 1B). Alignment of the Drosophila Pi3K59F sequence with its human homolog showed evolutionary conservation of these class III PI3K proteins, which demonstrated $64 \%$ identity and $79 \%$ similarity (Figure 1C). Moreover, both sequences contain C2, PIK, and kinase catalytic domains. ELM prediction identified an NES, a nuclear localization signal, several Atg8-binding motifs, and a di-arginine endoplasmic reticulum retention motif in the Drosophila sequence (Dinkel et al., 2016).

\section{Inhibition of Atg6 or Pi3K59F in the developing Drosophila eye results in different eye phenotypes}

The directed inhibition of Atg6 or Pi3K59F in the developing eye caused significant disruption of the ommatidial array, and whereas inhibition of Atg6 resulted in a higher mean number of ommatidia, that of Pi3K59F had the opposite effect (Figure 2A, I-III). The suppression of these two autophagy genes in the developing eye greatly disrupted the ommatidia (Figure 2B), implying a role for these proteins in normal eye development.

Co-expression of Atg6-RNAi or Pi3K59F-RNAi and $\alpha$-synuclein in the eye intensified developmental eye defects (Figure 2A, IV-VI). Disruption of the ommatidial array was pronounced, and the mean number of ommatidia was reduced (Figure 2C). These results indicate that inhibition of Atg6 or Pi3K59F in the eye, coupled with the toxic effects of $\alpha$-synuclein, enhances developmental abnormalities in this organ.

In contrast, co-expression of Atg6-RNAi or Pi3K59F-RNAi and Buffy in developing eye resulted in a slight increase in the number of ommatidia and a marked decrease in disruption of the ommatidial array (Figure 2A and D, VII-IX).

Genetics and Molecular Research 15 (4): gmr15048953 


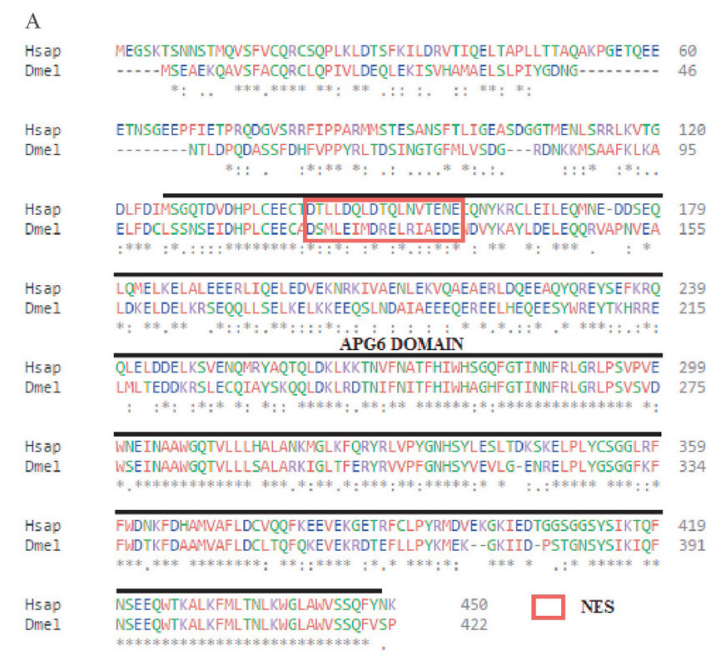

B

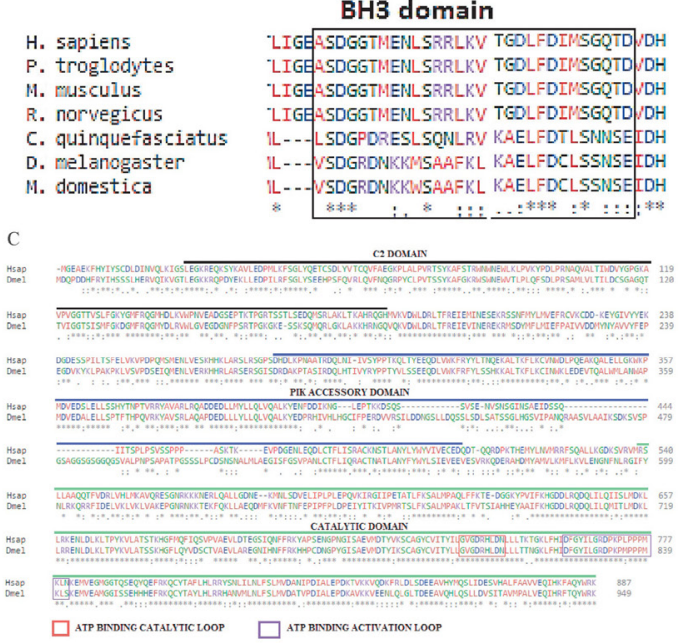

Figure 1. Autophagy proteins Atg6 and Pi3K59F are evolutionarily conserved. A. Multiple-sequence alignment of human Beclin 1 and its Drosophila melanogaster homologue Atg6 reveals conservation of the APG6/Vps30 domain important for initiation of autophagy, and presence of a nuclear export signal (NES). Hsap = Homo sapiens NP_003757.1, Dmel = D. melanogaster NP_651209.1. B. Drosophila sequence contains a putative BH3 domain, which is conserved with the exception of a small number of amino acid substitutions, as demonstrated by Clustal Omega (Goujon et al., 2010; Sievers et al., 2011) multiple-sequence alignment of the Drosophila Atg6 protein sequence with that of mammalian and insect homologues. H. sapiens $=H$. sapiens NP 003757.1, P. troglodytes $=$ Pan troglodytes XP_511522.2, M. musculus $=$ Mus musculus NP_062530.2, R. norvegicus $=$ Rattus norvegicus NP 001029289.1, C. quinquefasciatus $=$ Culex quinquefasciatus XP 001861442.1, D. melanogaster $=D$. melanogaster NP_651209.1, M. domestica = Musca domestica XP_005189450.1. C. Pi3K59F is highly conserved, with sequences from both organisms containing C2, PIK accessory, and kinase catalytic domains. Hsap $=H$. sapiens NP 002638.2, Dmel $=D$. melanogaster NP 477133.1. Domains were identified using the National Center for Biotechnology Information Conserved Domain Database (Marchler-Bauer et al., 2015). Asterisks indicate identical residues, colons indicate conserved substitutions, and periods indicate semi-conserved substitutions. Colors represent the chemical properties of each amino acid, as follows: red = small hydrophobic (including aromatic), blue $=$ acidic, magenta $=$ basic, green $=$ basic with hydroxyl or amine groups .

Genetics and Molecular Research 15 (4): gmr15048953 
A

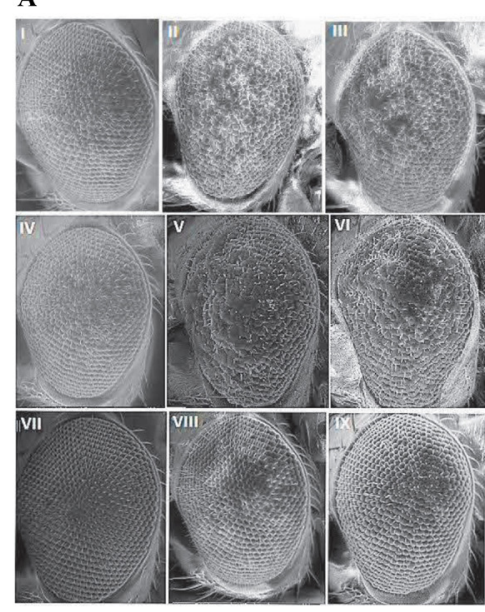

B
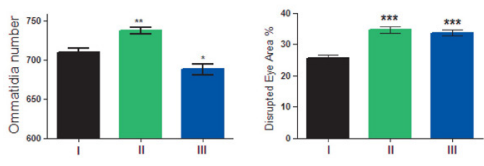

C

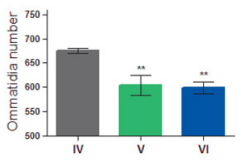

D

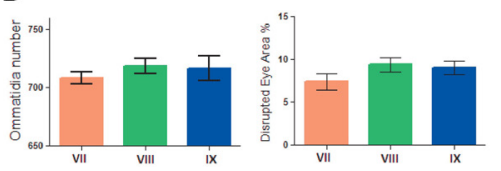

Figure 2. Conditional expression of Atg6-RNAi or Pi3K59F-RNAi in the developing Drosophila eye results in different phenotypes. A. Scanning electron micrographs of developing eyes in which Atg6 or Pi3K59F expression has been suppressed in isolation, or in the context of $\alpha$-synuclein or Buffy overexpression; (I) GMR-Gal4/UASlacZ, (II) GMR-Gal4/UAS-Atg6-RNAi, (III) GMR-Gal4/UAS-Pi3K59F-RNAi, (IV) UAS- - -synuclein; GMR-Gal4/ UAS-lacZ, (V) UAS- $\alpha$-synuclein; GMR-Gal4/UAS-Atg6-RNAi, (VI) UAS- $\alpha$-synuclein; GMR-Gal4/UAS-Pi3K59FRNAi, (VII) UAS-Buffy; GMR-Gal4/UAS-lacZ, (VIII) UAS-Buffy; GMR-Gal4/UAS-Atg6-RNAi, (IX) UAS-Buffy; GMR-Gal4/UAS-Pi3K59F-RNAi. B. Biometric analysis shows a significant difference in the number of ommatidia and disrupted area of the ommatidial array, with Atg6-RNAi and Pi3K59F-RNAi flies demonstrating higher and lower mean numbers of ommatidia, respectively, compared to control flies. C. Inhibition of Atg6 or Pi3K59F combined with $\alpha$-synuclein overexpression results in lower ommatidial count and larger disrupted eye areas than those observed in the control group. D. Co-expression of Buffy and Atg6-RNAi or Pi3K59F-RNAi in the eye did not significantly increase the mean number of ommatidia and the disrupted eye area compared to the control group that expressed both Buffy and the benign lacZ transgene. Comparisons were performed by one-way analysis of variance and the Dunnett multiple-comparison test $(\mathrm{P}<0.05 ; \mathrm{N}=10)$. Error bars show standard error of the mean. $* \mathrm{P}<0.05$, $* * \mathrm{P}<0.01$, and $* * * \mathrm{P}<0.001$.

\section{Inhibition of Atg6 or Pi3K59F in neurons replicates PD phenotypes}

Inhibition of Atg6 or Pi3K59F in Ddc-Gal4-expressing neurons by RNAi under control of the $D d c$-Gal4 transgene decreased fly survival (Figure 3A). The median lifespans of Atg6-RNAi and Pi3K59F-RNAi flies were 58 and 64 days, compared to 73 days for the control group. The directed inhibition of Atg6 or Pi3K59F in these neurons impaired climbing ability, as indicated by comparison of the climbing curves and associated $95 \%$ CIs (Figure 3B). Thus, neuronal inhibition of Atg6 or Pi3K59F appears to decrease Drosophila longevity and climbing ability; normal levels of the corresponding proteins are therefore required for the correct functioning of neurons.

\section{Inhibition of Atg6 or Pi3K59F in the $\alpha$-synuclein-induced PD model alters survival but not climbing ability}

Expression of $\alpha$-synuclein in DA neurons impaired the climbing ability of flies and shortened their lifespan. In comparison, inhibition of Atg6 or Pi3K59F in the neurons of flies expressing $\alpha$-synuclein lowered lifespan but did not affect locomotor ability (Figure 4A and 
B). Atg6-RNAi flies had a median lifespan of 56 days, as did Pi3K59F-RNAi flies, compared to the control group that had a median of 62 days. Non-linear fitting of curves with $95 \% \mathrm{CIs}$ revealed that climbing indices did not significantly differ between the Atg6-RNAi, Pi3K59F$R N A i$, and control groups, although slightly lower values were observed in the former two than in the latter. This indicates that inhibition of these autophagy genes somewhat modifies the phenotype induced by expression of $\alpha$-synuclein in Ddc-Gal4-expressing Drosophila neurons.
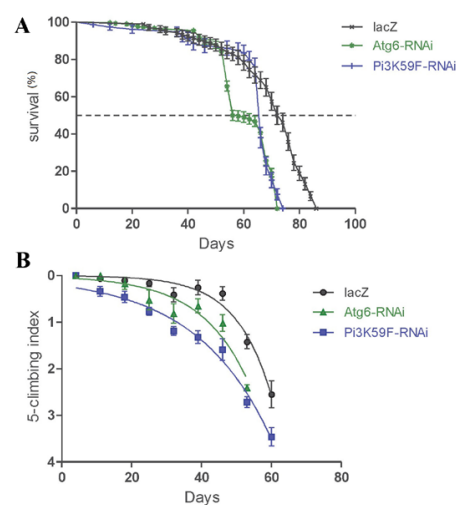

Figure 3. Inhibition of Atg6 or Pi3K59F in neurons reproduces Parkinson's disease-like phenotypes. A. Directed inhibition of Atg6 or Pi3K59F in Ddc-Gal4-expressing neurons decreases survival compared to control flies overexpressing the benign UAS-lacZ transgene. Fly genotypes were Ddc-Gal4/UAS-lacZ, Ddc-Gal4/UAS-Atg6$R N A i$, and $D d c$-Gal4/UAS-Pi3K59F-RNAi. Longevity is reported as the percentage of surviving flies $[\mathrm{P}<0.05$, determined by the log-rank (Mantel-Cox) test; $\mathrm{N} \geq 200$ ]. B. Inhibition of Atg6 or Pi3K59F in these neurons significantly decreases climbing ability, as determined by non-linear fitting of the climbing curves and comparisons of $95 \%$ confidence intervals. Fly genotypes were Ddc-Gal4/UAS-lacZ, Ddc-Gal4/UAS-Atg6-RNAi, and Ddc-Gal4/ $U A S-P i 3 K 59 F-R N A i$. Error bars indicate standard error of the mean $(\mathrm{N}=50)$.

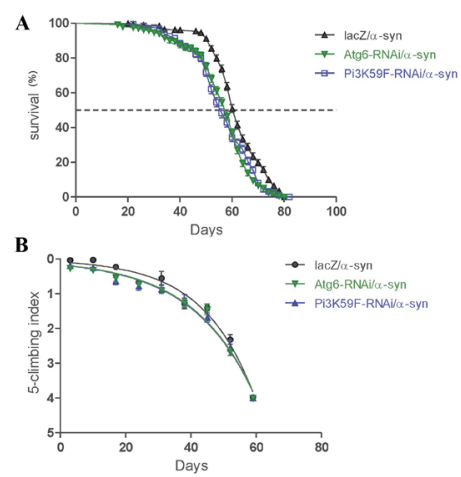

Figure 4. Inhibition of Atg6 or Pi3K59F in an $\alpha$-synuclein-induced model of Parkinson's disease (PD) slightly alters survival, but not climbing ability. A. Inhibition of Atg6 or Pi3K59F together with $\alpha$-synuclein ( $\alpha$-syn) overexpression in $D d c$-Gal4-expressing neurons slightly decreases median lifespan compared to control flies. Fly genotypes were UAS- $\alpha$-synuclein; Ddc-Gal4/UAS-lacZ, UAS- $\alpha$-synuclein; Ddc-Gal4/UAS-Atg6-RNAi, and UAS- $\alpha$-synuclein; DdcGal4/UAS-Pi3K59F-RNAi. Longevity is reported as the percentage of surviving flies $[\mathrm{P}<0.05$, determined by log-rank (Mantel-Cox) test; $\mathrm{N} \leq 200]$. B. Expression of Atg6-RNAi or Pi3K59F-RNAi in the $\alpha$-synuclein-induced model of PD did not significantly affect age-dependent loss of climbing ability compared to the control group. Fly genotypes were UAS- $\alpha$-synuclein; Ddc-Gal4/UAS-lacZ, UAS- $\alpha$-synuclein; Ddc-Gal4/UAS-Atg6-RNAi, and UAS- $\alpha$-synuclein; Ddc-Gal4/UAS-Pi3K59F-RNAi. Error bars indicate standard error of the mean $(\mathrm{N}=50)$.

Genetics and Molecular Research 15 (4): gmr15048953 


\section{Buffy overexpression enhances Atg6-RNAi- and suppresses Pi3K59F-RNAi-induced phenotypes}

Co-expression of Atg6-RNAi or Pi3K59F-RNAi and the pro-survival Bcl-2 Drosophila homolog Buffy enhanced the phenotype caused by reduction of Atg6 expression, and rescued loss of Pi3K59F expression (Figure 5). Buffy overexpression combined with RNAi inhibition of Atg6 reduced survival to a median lifespan of 46 days, compared to 66 days for control flies (Figure 5A). The impaired climbing ability exhibited by the Atg6-RNAi flies was amplified as determined by non-linear fitting of climbing curves and 95\%CIs (Figure 5B). This may be due to interaction between Buffy and the BH3 domain of Atg6, restricting the latter's ability to initiate autophagy. Interestingly, overexpression of Buffy in Pi3K59F-RNAi flies suppressed the reduced survival and climbing ability resulting from Pi3K59F inhibition. The median lifespan of these flies (66 days) was the same as that of the control flies (Figure 5A). In addition, their climbing ability improved to normal levels (Figure 5B).

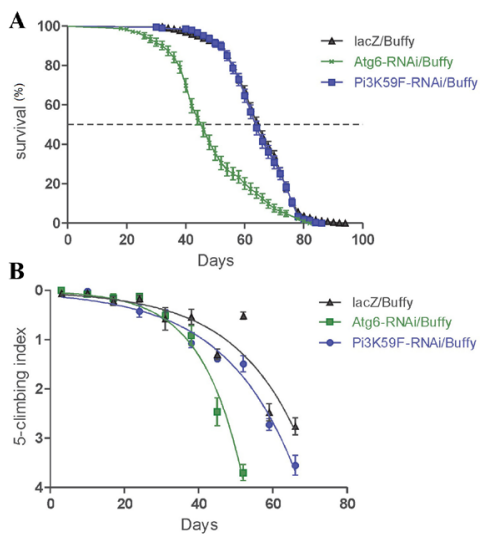

Figure 5. Buffy overexpression enhances and suppresses phenotypes induced by Atg6-RNAi and Pi3K59F-RNAi, respectively. A. Co-expression of Buffy and Atg6-RNAi exacerbates the decrease in survival caused by suppression of Atg6, whereas co-expression of Buffy and Pi3K59F-RNAi negates the lowered median survival resulting from reduced Pi3K59F expression. Fly genotypes were UAS-Buffy; Ddc-Gal4/UAS-lacZ, UAS-Buffy; Ddc-Gal4/UASAtg6-RNAi, and UAS-Buffy; Ddc-Gal4/UAS-Pi3K59F-RNAi. B. Co-expression of Atg6-RNAi and Buffy in DdcGal4-expressing neurons worsens age-dependent loss of climbing ability. In contrast, co-expression of Pi3K59F$R N A i$ and Buffy suppresses age-dependent loss of climbing ability compared to flies expressing Pi3K59F-RNAi alone. Fly genotypes were UAS-Buffy; Ddc-Gal4/UAS-lacZ, UAS-Buffy; Ddc-Gal4/UAS-Atg6-RNAi, and UASBuffy; Ddc-Gal4/UAS-Pi3K59F-RNAi. Error bars indicate standard error of the mean $(\mathrm{N}=50)$.

\section{DISCUSSION}

Biometric analysis following inhibition of Atg6 or Pi3K59F showed significant differences in Drosophila eye phenotypes. Reduced Atg6 expression significantly increased the number of ommatidia and the size of the disrupted eye area. However, when Pi3K59F was inhibited, fewer ommatidia were observed, along with a high degree of disruption to the ommatidial array. Although these two autophagy proteins are known to be members of the same complex, it is possible that when each is conditionally inhibited, they exert different effects on the developing eye. Altered Atg6 or Pi3K59F expression in the $\alpha$-synuclein-

Genetics and Molecular Research 15 (4): gmr15048953 
expressing developmental eye defect model resulted in an enhanced phenotype. Both RNAi transgenes caused reduced numbers of ommatidia and ommatidial disarray. It is interesting that inhibition of Atg6 or Pi3K59F in an $\alpha$-synuclein-overexpression background enhanced the eye development phenotype, a trend that we did not observe with Ddc-Gal4-expressing neurons. The toxic effects of $\alpha$-synuclein in the developing eye have been well documented (Feany and Bender, 2000), and demonstrate an additive effect with eye phenotype worsening under additional assaults (M'Angale and Staveley, 2012). It seems therefore that inhibition of the autophagy genes Atg6 or Pi3K59F in tandem with $\alpha$-synuclein overexpression produces severe eye disruption. Overexpression of Buffy in Drosophila is known to suppress stress phenotypes and improve the "healthspan" of flies (Quinn et al., 2003; M'Angale and Staveley, 2016). Notably, inhibition of Pi3K59F in the developing eye resulted in fewer ommatidia, whereas co-expression of Pi3K59F-RNAi and Buffy raised the number of ommatidia and improved the degeneration of the eye tremendously. Expression of Atg6-RNAi in the developing eye caused higher numbers of ommatidia coupled by high levels of ommatidial degeneration, and its coexpression with Buffy resulted in reduced ommatidial counts and lower disrupted eye areas.

Conditional expression of Atg6-RNAi or Pi3K59F-RNAi in Drosophila neurons under the direction of the $D d c$-Gal4 transgene reduced survival and increased the age-dependent loss of climbing ability, phenotypic attributes that are strongly associated with Drosophila models of PD. The reduction in Atg6 and Pi3K59F activity in these neurons may be detrimental to the health of these flies, manifesting as PD-like symptoms. Atg6 and Pi3K59F are involved in the nucleation phase of autophagy during autophagosome formation (Levine and Kroemer, 2008). Inhibition of these two important autophagy genes may lead to decreased autophagic activity that appears to disrupt neuronal function.

Flies in which $\alpha$-synuclein is expressed in Ddc-Gal4-expressing neurons demonstrate impaired locomotor function. In previous studies, $\alpha$-synuclein accumulation in LBs and Lewy neurites has been attributed to failure of the ubiquitin proteasome degradation system (Auluck et al., 2002). However, recent investigations have shown that $\alpha$-synuclein may be degraded via the lysosomal pathway, in particular, by macroautophagy and chaperone-mediated autophagy (CMA; Xilouri and Stefanis, 2011; Jiang and Mizushima, 2014). Co-expression of Atg6-RNAi or Pi3K59F-RNAi and $\alpha$-synuclein slightly reduced longevity and climbing ability compared to flies overexpressing $\alpha$-synuclein alone. Inhibition of autophagy leads to the accumulation of $\alpha$-synuclein, demonstrating an important role for this process in normal $\alpha$-synuclein turnover (Webb et al., 2003). Post-translational modifications of $\alpha$-synuclein interfere with its degradation and that of other proteins by CMA (Martinez-Vicente et al., 2008), and inhibit autophagosome formation at an early stage (Winslow et al., 2010). These findings indicate a compromised autophagy system; therefore, $\alpha$-synuclein toxicity and $\alpha$-synuclein-induced phenotypes are only slightly enhanced by a further reduction in autophagic activity. Such observations highlight the complexity of PD pathogenesis, and demonstrate the involvement of numerous failed cellular mechanisms.

Members of the $\mathrm{Bcl}-2$ family bind $\mathrm{Atg} 6$, a $\mathrm{BH} 3$-only protein, in a multimeric complex involved in the vesicle nucleation stage of autophagosome formation. Discovery of the BH3 domain in Atg6, a binding site allowing interaction with the anti-apoptotic Bcl-2 proteins and required for Atg6 inhibition, revealed that Bcl-2 proteins not only regulate apoptosis, but also have an anti-autophagic function (Sinha and Levine, 2008). Bcl-2 proteins appear to play a crucial role in maintaining autophagic homeostasis, as weakening their interaction with Atg6 by its phosphorylation promotes autophagy (Zalckvar et al., 2009). Atg6 contains a Pi3K59F

Genetics and Molecular Research 15 (4): gmr15048953 
binding site, and it is this interaction that leads to the production of the phosphatidylinositol3-phosphate pivotal to the nucleation process (Furuya et al., 2005). Overexpression of the $B c l-2$ homolog Buffy, together with Atg6-RNAi, resulted in lower survival and climbing scores compared to flies expressing Atg6-RNAi alone. Notably, however, Buffy overexpression rescued the effects of inhibiting Pi3K59F. Taken together, these results indicate that Buffy magnifies Atg6-RNAi-induced phenotypes and suppresses those resulting from Pi3K59F-RNAi. Since Bcl-2 proteins have been shown to block Atg6-dependent autophagy by inhibiting the formation of the Atg6/Pi3K59F complex (Pattingre et al., 2005), we are inclined to speculate that Buffy plays a similar role in D. melanogaster. Excessive autophagy leads to cell death; therefore, Buffy may act to balance autophagic activity via the Atg6 pathway involved in the elimination of dysfunctional mitochondria, counteracting PD-like symptoms in Drosophila. The accumulation of $\alpha$-synuclein promotes excessive autophagy levels, leading to removal of mitochondria, the depletion of which from most neurons may interrupt synaptic function.

\section{CONCLUSIONS}

Inhibition of either Atg6 or Pi3K59F resulted in reduced longevity and impaired locomotor function, possibly representing a novel model of PD. It is important to establish other roles for these proteins, besides those in autophagy, so as to further understand their functions. Buffy, similar to most pro-survival Bcl-2 proteins, demonstrates anti-autophagic effects that enhance and suppress phenotypes associated with inhibition of Atg6 or Pi3K59F, respectively. The mechanism by which Buffy affects this response remains unclear. However, it may involve interaction with the putative BH3 domain in Atg6, disrupting formation of the Atg6/Pi3K59F complex that is key to the initiation of autophagy. Further studies are required to elucidate the roles of Atg6, Pi3K59F, and Buffy in the autophagy-lysosome pathway.

\section{Conflicts of interest}

The authors declare no conflict of interest.

\section{ACKNOWLEDGMENTS}

P.G. M'Angale was partially funded by Department of Biology Teaching Assistantships and a School of Graduate Studies Fellowship from Memorial University of Newfoundland. B.E. Staveley was funded by a Natural Sciences and Engineering Research Council of Canada (NSERC) Discovery Grant.

\section{REFERENCES}

Auluck PK, Chan HY, Trojanowski JQ, Lee VM, et al. (2002). Chaperone suppression of alpha-synuclein toxicity in a Drosophila model for Parkinson's disease. Science 295: 865-868.http://dx.doi.org/10.1126/science.1067389

Chang YY and Neufeld TP (2010). Autophagy takes flight in Drosophila. FEBS Lett. 584: 1342-1349. http://dx.doi. org/10.1016/j.febslet.2010.01.006

Chinta SJ, Mallajosyula JK, Rane A and Andersen JK (2010). Mitochondrial $\alpha$-synuclein accumulation impairs complex I function in dopaminergic neurons and results in increased mitophagy in vivo. Neurosci. Lett. 486: 235-239. http:// dx.doi.org/10.1016/j.neulet.2010.09.061

Cuervo AM, Stefanis L, Fredenburg R, Lansbury PT, et al. (2004). Impaired degradation of mutant alpha-synuclein by chaperone-mediated autophagy. Science 305: 1292-1295.http://dx.doi.org/10.1126/science.1101738

Genetics and Molecular Research 15 (4): gmr15048953 
Dehay B, Vila M, Bezard E, Brundin P, et al. (2016). Alpha-synuclein propagation: New insights from animal models. Mov. Disord. 31: 161-168. http://dx.doi.org/10.1002/mds.26370

Dinkel H, Van Roey K, Michael S, Kumar M, et al. (2016). ELM 2016 - data update and new functionality of the eukaryotic linear motif resource. Nucleic Acids Res. 44 (D1): D294-D300. http://dx.doi.org/10.1093/nar/gkv1291

Esteves AR, Arduíno DM, Silva DF, Oliveira CR, et al. (2011). Mitochondrial dysfunction: the road to alpha-synuclein oligomerization in PD. Parkinsons Dis. 2011: 693761.http://dx.doi.org/10.4061/2011/693761

Feany MB and Bender WW (2000). A Drosophila model of Parkinson's disease. Nature 404: 394-398. http://dx.doi. org $/ 10.1038 / 35006074$

Forno LS (1996). Neuropathology of Parkinson's disease. J. Neuropathol. Exp. Neurol. 55: 259-272. http://dx.doi. org/10.1097/00005072-199603000-00001

Freeman M (1996). Reiterative use of the EGF receptor triggers differentiation of all cell types in the Drosophila eye. Cell 87: 651-660. http://dx.doi.org/10.1016/S0092-8674(00)81385-9

Furuya N, Yu J, Byfield M, Pattingre S, et al. (2005). The evolutionarily conserved domain of Beclin 1 is required for Vps34 binding, autophagy and tumor suppressor function. Autophagy 1: 46-52.http://dx.doi.org/10.4161/auto.1.1.1542

Goujon M, McWilliam H, Li W, Valentin F, et al. (2010). A new bioinformatics analysis tools framework at EMBL-EBI. Nucleic Acids Res. 38: W695-9. http://dx.doi.org/10.1093/nar/gkq313

Jiang P and Mizushima N (2014). Autophagy and human diseases. Cell Res. 24: 69-79. http://dx.doi.org/10.1038/ cr.2013.161

Juhász G, Hill JH, Yan Y, Sass M, et al. (2008). The class III PI(3)K Vps34 promotes autophagy and endocytosis but not TOR signaling in Drosophila. J. Cell Biol. 181: 655-666.http://dx.doi.org/10.1083/jcb.200712051

la Cour T, Kiemer L, Mølgaard A, Gupta R, et al. (2004). Analysis and prediction of leucine-rich nuclear export signals. Protein Eng. Des. Sel. 17: 527-536. http://dx.doi.org/10.1093/protein/gzh062

Levine B and Kroemer G (2008). Autophagy in the pathogenesis of disease. Cell 132: 27-42. http://dx.doi.org/10.1016/j. cell.2007.12.018

Li H, Chaney S, Roberts IJ, Forte M, et al. (2000). Ectopic G-protein expression in dopamine and serotonin neurons blocks cocaine sensitization in Drosophila melanogaster. Curr. Biol. 10: 211-214. http://dx.doi.org/10.1016/S0960$\underline{\text { 9822(00)00340-7 }}$

Lindqvist LM, Heinlein M, Huang DC and Vaux DL (2014). Prosurvival Bcl-2 family members affect autophagy only indirectly, by inhibiting Bax and Bak. Proc. Natl. Acad. Sci. USA 111: 8512-8517. http://dx.doi.org/10.1073/ pnas. 1406425111

M'Angale PG and Staveley BE (2012). Effects of $\alpha$-synuclein expression in the developing Drosophila eye. Drosoph. Inf. Serv. 95: 85-89.

M'Angale PG and Staveley BE (2016). The Bcl-2 homologue Buffy rescues $\alpha$-synuclein-induced Parkinson disease-like phenotypes in Drosophila. BMC Neurosci. 17: 24. http://dx.doi.org/10.1186/s12868-016-0261-z

Marchler-Bauer A, Derbyshire MK, Gonzales NR, Lu S, et al. (2015). CDD: NCBI's conserved domain database. Nucleic Acids Res. 43: D222-D226. http://dx.doi.org/10.1093/nar/gku1221

Martinez-Vicente M, Talloczy Z, Kaushik S, Massey AC, et al. (2008). Dopamine-modified alpha-synuclein blocks chaperone-mediated autophagy. J. Clin. Invest. 118: 777-788.

McPhee CK and Baehrecke EH (2009). Autophagy in Drosophila melanogaster. Biochim. Biophys. Acta 1793: 1452-1460. http://dx.doi.org/10.1016/j.bbamcr.2009.02.009

Pattingre S, Tassa A, Qu X, Garuti R, et al. (2005). Bcl-2 antiapoptotic proteins inhibit Beclin 1-dependent autophagy. Cell 122: 927-939. http://dx.doi.org/10.1016/j.cell.2005.07.002

Perrett RM, Alexopoulou Z and Tofaris GK (2015). The endosomal pathway in Parkinson's disease. Mol. Cell. Neurosci. 66 (Pt A): 21-28. http://dx.doi.org/10.1016/j.men.2015.02.009

Polymeropoulos MH, Lavedan C, Leroy E, Ide SE, et al. (1997). Mutation in the $\alpha$-synuclein gene identified in families with Parkinson's disease. Science 276: 2045-2047. http://dx.doi.org/10.1126/science.276.5321.2045

Quinn L, Coombe M, Mills K, Daish T, et al. (2003). Buffy, a Drosophila Bcl-2 protein, has anti-apoptotic and cell cycle inhibitory functions. EMBO J. 22: 3568-3579. http://dx.doi.org/10.1093/emboj/cdg355

Schneider CA, Rasband WS and Eliceiri KW (2012). NIH Image to ImageJ: 25 years of image analysis. Nat. Methods 9: 671-675. http://dx.doi.org/10.1038/nmeth.2089

Sievers F, Wilm A, Dineen D, Gibson TJ, et al. (2011). Fast, scalable generation of high-quality protein multiple sequence alignments using Clustal Omega. Mol. Syst. Biol. 7: 539.http://dx.doi.org/10.1038/msb.2011.75

Sinha S and Levine B (2008). The autophagy effector Beclin 1: a novel BH3-only protein. Oncogene 27 (Suppl 1): S137-S148.http://dx.doi.org/10.1038/onc.2009.51

Staveley BE (2014). Drosophila models of Parkinson disease. In: Movement disorders: genetics and models (LeDoux MS, ed.). Academic Press, Cambridge, 345-354.

Genetics and Molecular Research 15 (4): gmr15048953 
Staveley BE, Phillips JP and Hilliker AJ (1990). Phenotypic consequences of copper-zinc superoxide dismutase overexpression in Drosophila melanogaster. Genome 33: 867-872. http://dx.doi.org/10.1139/g90-130

Todd AM and Staveley BE (2004). Novel assay and analysis for measuring climbing ability in Drosophila. Drosoph. Inf. Serv. 87: 101-107.

Todd AM and Staveley BE (2012). Expression of Pink1 with $\alpha$-synuclein in the dopaminergic neurons of Drosophila leads to increases in both lifespan and healthspan. Genet. Mol. Res. 11: 1497-1502. http://dx.doi.org/10.4238/2012. May.21.6

Webb JL, Ravikumar B, Atkins J, Skepper JN, et al. (2003). $\alpha$-Synuclein is degraded by both autophagy and the proteasome. J. Biol. Chem. 278: 25009-25013. http://dx.doi.org/10.1074/jbc.M300227200

Winslow AR, Chen CW, Corrochano S, Acevedo-Arozena A, et al. (2010). $\alpha$-Synuclein impairs macroautophagy: implications for Parkinson's disease. J. Cell Biol. 190: 1023-1037.http://dx.doi.org/10.1083/jcb.201003122

Xilouri M and Stefanis L (2011). Autophagic pathways in Parkinson disease and related disorders. Expert Rev. Mol. Med. 13: e8.http://dx.doi.org/10.1017/S1462399411001803

Xilouri M and Stefanis L (2015). Chaperone mediated autophagy to the rescue: A new-fangled target for the treatment of neurodegenerative diseases. Mol. Cell. Neurosci. 66 (Pt A): 29-36. http://dx.doi.org/10.1016/j.mcn.2015.01.003

Zalckvar E, Berissi H, Mizrachy L, Idelchuk Y, et al. (2009). DAP-kinase-mediated phosphorylation on the BH3 domain of beclin 1 promotes dissociation of beclin 1 from Bcl-XL and induction of autophagy. EMBO Rep. 10: 285-292. http:// dx.doi.org/10.1038/embor.2008.246

Zirin J and Perrimon N (2010). Drosophila as a model system to study autophagy. Semin. Immunopathol. 32: 363-372. http://dx.doi.org/10.1007/s00281-010-0223-y

Genetics and Molecular Research 15 (4): gmr15048953 